\title{
Battery health and performance monitoring system: a closer look at state of health (SoH) assessment methods of a lead-acid battery
}

\author{
D. Selvabharathi, N. Muruganantham \\ Department of EEE, Periyar Maniammai Institute of Science \& Technology, India
}

\begin{tabular}{l}
\hline Article Info \\
\hline Article history: \\
Received Aug 6, 2019 \\
Revised Oct 8, 2019 \\
Accepted Oct 23, 2019 \\
\hline Keywords: \\
Aging mechanism \\
Battery \\
Lead-acid \\
State of charge \\
State of health \\
\hline
\end{tabular}

\begin{abstract}
Batteries are one of the most compact and reliable sources of sustainable energy. Lead-Acid batteries are the battery-powered sort of batteries concocted during the 1980s. The significant utilization of lead-acid battery is in beginning, lighting and start frameworks of vehicles. To guarantee the health and to dodge potential disappointments of a battery it is important to examine its Territory of health precisely. This examination expects to give efficiently evaluating the accessible writing on the condition of health estimation techniques. This study focuses on many factors and provides a suggestion for the defended battery manufacturing process. This study provides increasing efforts toward the advancement of battery interms of specific power, energy density, durability, invulnerability, economics, and performance in various applications.
\end{abstract}

Copyright $@ 2020$ Institute of Advanced Engineering and Science. All rights reserved.

\section{Corresponding Author:}

D. Selvabharathi,

Department of EEE,

Periyar Maniammai Institute of Science \& Technology,

Thanjavur, India.

Email: dsbsrm@gmail.com

\section{INTRODUCTION}

A battery is an electro-chemical energy storage device; the battery provides DC electrical energy during the discharging process, which changes the charging balance between chemically active materials that are central to the electro-chemical reactions [1]. The charge process coverts the DC Electrical energy into stored chemical energy, during the discharge process battery again converts the Stored chemical energy into electric DC [2]. For any batteries the manufacturer used to specify given number of cycles for charging and discharging. A battery cannot have well defined life span and the health of the battery depends on conditions of environment and also usage patterns [3].

A lead corrosive-battery achieves the finish of life when the dynamic material has been devoured on the positive matrices; nickel-based batteries lose execution due to erosion [4]. Lithium-particle weakens after some time when the exchange of particles backs off because of the development of layers on the cathodes [5].

Battery makers know about execution misfortune after some time, and there is a conscious quietness on battery maturing brought about by the limit blur. Runtimes are constantly assessed with an ideal battery conveying 100 percent limit. This is a flashing condition that possibly applies when the battery is new [6]. By and large acknowledged test methodology for checking State of Health have not yet been building up for batteries and even reviewers doing quality control timid far from such managing and just look at the external appearance, condition of SoH for the most part overlooked [7]. This enables weak batteries to cover up easily among their stronger peers. The oversight is to a limited extent associated with an absence of battery test gadgets that can dependably survey a battery on the fly [8]. 
Complications in Current System:

a) Battery state and efficiency is difficult to notice

b) No prior Battery failure identification

c) The system may get damaged due to Emergency replacement

d) Battery life decreases due to improper maintenance.

e) Operating cost of the battery also will get increased due to improper maintenance.

f) A major problem in monitoring the battery is the unavailability of data for recognition and analyzing the fault

Many research related articles presented classifications of different health conditions related to $\mathrm{SoH}$ and each battery has individual characteristics [9]. In order to get better battery management, different battery SoH assessment methods based on experimental analysis are reviewed.

\section{EVALUATING THE STATE OF HEALTH}

The important part in assessing $\mathrm{SoH}$ is to differentiate the useful life remaining for a battery and health state of a battery. Life cycle of a battery is characterized dependent on sort of battery, standard and material used by battery manufacturer in terms of number of cycles supported [12].

The important part in assessing $\mathrm{SoH}$ is to differentiate the useful life remaining for a battery and health condition of a battery. Life process of a battery is defined by battery type, standard and material used by battery manufacturer in terms of number of cycles supported $[13,14]$. State of Health is defined as representing the health condition and performance comparison of old battery with the new one of same type with same ratings [15]. Health condition is nothing but the ratio of maximum discharge power to the rated discharge power of the specific battery.

$$
\text { State of Health }(\mathrm{SoH})=\frac{\text { Maximum Discharge Power }}{\text { Rated Discharge Power }} \times 100 \%
$$

The main challenge in State of Health estimation is to determine the Maximum discharge power accurately. Figure 1 summarizes the representation of Battery SoH.

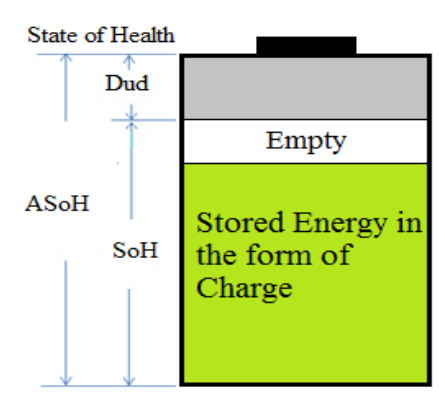

Figure 1. Representation of battery SoH

Definition:

SoH : State of Health

ASoH :Absolute State of health of a new battery

$\mathrm{RSoH}$ :Relative state of health to available capacity

Continuous decline in the performance of battery is produced by constant chemical responses and this response conduce fading of capacity and devaluation in the rest of their useful life. Although determining Sate of Charge (SoC) is possible by measuring time and current [16].

\subsection{Fundamentals in Testing the Batteries}

For finding the state of health there is no exact method. There are various features can be used to determine SoH such as

a) Based on Capacity

b) Based on Internal resistance of the battery

c) Based on Self-Discharging quantity of the battery 
While manufacturing the battery the manufacturer/designer cannot able to anticipate the health of the battery before a device enters service. Health management is the important parameter for finding the $\mathrm{SoH}$ value of the battery for different times in battery life cycles $[17,18]$. The $\mathrm{SoH}$ of the battery can be changed with the following parameters internal resistance, battery capacity, usage time etc. A change in the above parameter generally indicates another alternation to the usage customer. This will lead to reduce the battery performance in the form of temperature rise, continuous power loss, and passivation of electrodes, corrosion and gassing [19].

\subsection{Factors for Degradation and Aging Processes}

The factors of degradation are calculated based on effect of batteries life period. There are many reasons that cause health degradation of batteries mainly due to characteristics change in anode, cathode, electrolyte and other component used in the battery [20]. The aging process is done by two methods, first method is based on gradual degradation of battery over the operating time period and the second method is no noticeable symptoms of about degradation until a major problem occurred in battery performance. State of health estimation is always detects the first method of aging process since the gradual degradation is found by other battery parameters [21, 22].

Significant circumstances that accelerate battery degradation and life cycles are summarized as follows,

a) Discharge rate of battery is more

b) High Temperatures

c) Charging and discharging occurring in multiple cycles

d) Over Charging,

e) Over exhaust of the Battery

\section{MEASUREMENT METHODS}

The condition of the battery can be analyzed using two different methods such as direct measurement method and indirect measurement method [23]. Physical properties like terminal voltage and impedance of the battery can be calculated by direct measurement. Another method to estimate the SoH parameters are indirect measurement method which can be done by data analysis [24].

\subsection{Direct Measurement Method}

There are various methods have been proposed for the assessment of state of health in real time.

\subsubsection{Available Capacity based methods}

The capacity of the battery is nothing but the ability of a battery to store energy. The Battery State of Health can be determined by comparing nominal capacity with the available capacity as follows, where charge of the battery is the available capacity and nominal charge is the battery capacity, this nominal charge is nothing but the capacity of the battery when it is new [25]. Measuring capacity requires full charging and discharge of the battery; it is impractical situations in real time such as Electric Vehicles and Hybrid Electric Vehicles. Instead, this measurement can be calculated indirectly from measuring other parameters [26].

To calculate the available capacity and State of Health, the concept called genetic algorithms also used. A genetic algorithm are developed by the ways of thinking/basic truths/rules of natural selection and because of this it is used to solve the problems by imitating natural selection process by using recombination, selection of combinations, and change to get better solution to the problem. That's the reason for the genetic algorithm and an equivalent open circuit voltage model is used to estimate it through measuring the electrical storage device current and terminal voltage [27].

Nonlinear model through Ah (Ampere-hour) turnout are also used for estimation. The Ampere hour is the current turnout by the battery [28]. It can be used to estimate the battery ability by measuring the Ampere-hour turnout between low and high operating voltage limits using a non-linear function.

\subsubsection{Internal Resistance based methods}

There is a relationship between health of the battery and internal resistance of the battery. Therefore, this relationship has been utilized to estimate the Battery State of Health, which is given as follows,

When the battery is new online battery resistance is Ri and initial resistance is Ro. Figure 2 and Figure 3 shows the relationship between available capacity and internal resistance is given below. Dual extended Kalman filter is the measuring method used to estimate the battery resistance [29]. This resistance of the battery measure by using Kalman filter method is then combined with a battery aging model to estimate the State of Health. 


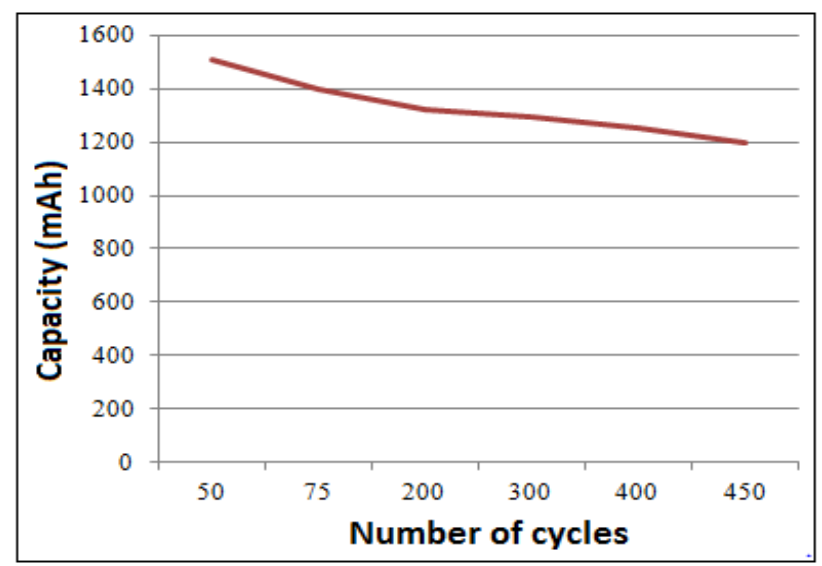

Figure 2. Relationship between capacities as part of cycling

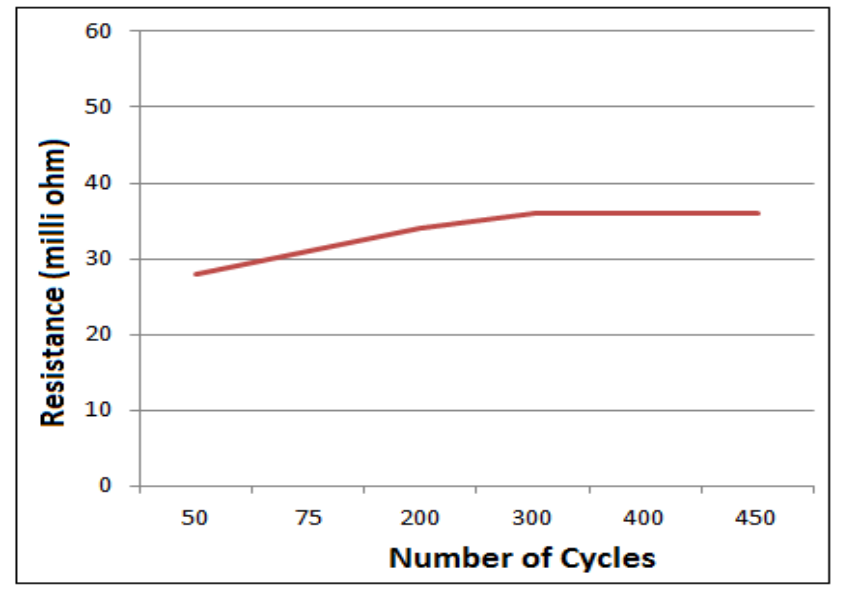

Figure 3. Relationship between resistances as part of cycling

Another major drawback in State of health estimation is temperature dependency of the battery; by compensating internal resistance we can able to fix this undesired dependency. This requires accessing the characteristics curve of the internal resistance over temperature for the battery cells before the battery is deployed. Further to temperature dependency, deviation between individual cells internal resistance in the battery causes further problems for accurate State of Health estimation [30].

\subsection{Indirect Measurement Methods}

An observational method based on experimental data is accepted to estimate the level of battery fading. The most popular method is Coulomb Counting method that allows SoH to be estimated by simple current integration over time. At regular intervals, it requires a calibration and cannot be done in real time [31].

Statistical method requires a large set of data to be effective as an analytical model. Using time series process, such as Auto Regressive Moving Average (ARMA), is a method that is mostly adaptive. This method only for one battery as each aging is different because it depends on the use of the battery. Another approach may consider the end-of-life criteria of the battery as a Weibull law Failure. This method however considers all different uses and conditions as a unique way to reduce the accuracy of the result.

\section{SUMMARY OF LEAD-ACID BATTERIES}

A lead-Acid battery keeps a study foothold as being rugged and dependable at a cost that is lower than maximum other chemistries [8]. The worldwide marketplace of lead-acid batteries is still developing however different systems are making inroads. Lead-acid batteries work fine for standby applications that 
require few deep discharge cycles and the starter battery fits this obligation properly [10]. Table 1 summarizes the characteristics of lead-acid batteries.

Table 1. Comparison of Various Lead-Acid Batteries

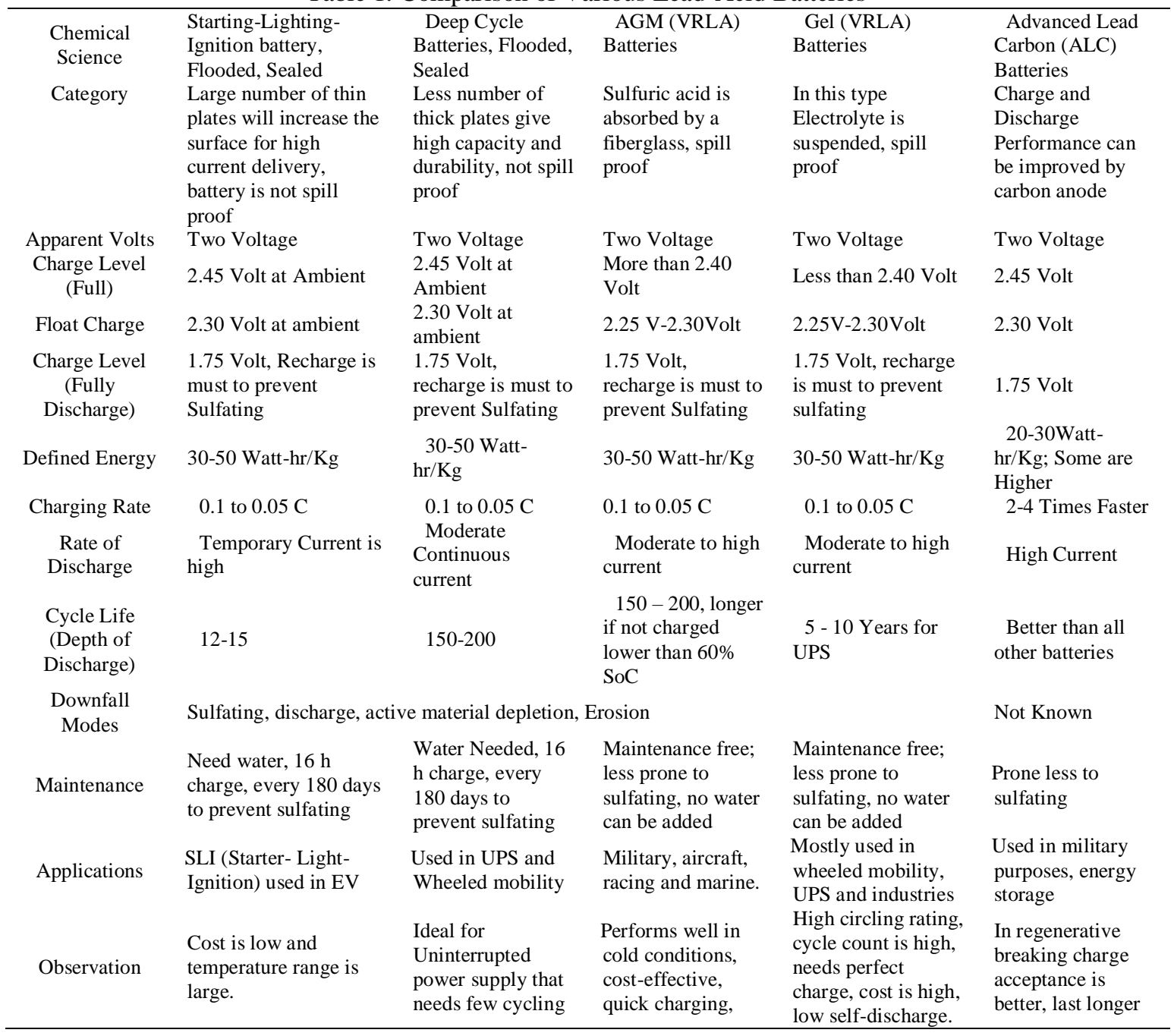

\section{CONCLUSION}

This study presented the Lead acid battery aging mechanism and reviewed various approaches present to SoH estimation. There are many methods explained and analyzed the aging processes. Each of estimation methods discussed in this study has the advantages as well as disadvantages characteristics. Direct measurement methods do not need a battery hypothesis because we are estimating the battery directly. Indirect methods like statistical methods are easily adjustable to different types of batteries and they can perform to give an aging diagnosis in real time. But this statistical method requires large amount of effective data. Therefore, obtaining a complete battery SoH diagnosis considering every aging factor and compatible with an application is still a major challenge.

\section{REFERENCES}

[1] RuiXiong, Linlin Li, JinpengTian, "Towards a smarter battery management system: A critical review on battery state of health monitoring methods”, Journal of Power Sources, Vol. 405, 30 November 2018, Pages 18-29

[2] TeodoraMurariu, CristianMorari, "Time-dependent analysis of the state-of-health for lead-acid batteries: An EIS study”, Journal of Energy Storage, Vol. 21, February 2019, Pages 87-93

[3] Ross Kerley, JiHoon Hyun, and Dong Sam Ha, "Automotive lead-acid battery state-of-health monitoring system", IECON 2015 - 41st Annual Conference of the IEEE Industrial Electronics Society, November 9-12, 2015

[4] Laifa Tao, Jian Ma, Yujie Cheng, Azadeh Noktehdan, Jin Chongf, Chen Lu, "A review of stochastic battery models and health management", Renewable and Sustainable Energy Reviews, Vol. 80, December 2017, Pages 716-732 
[5] Seyed Mohammad Rezvanizaniani*, Zongchang Liu, Yan Chen, Jay Lee, "Review and recent advances in battery health monitoring and prognostics technologies for electric vehicle (EV) safety and mobility", Journal of Power Sources, Vol. 256, 15 June 2014, Pages 110-124

[6] G.L. Plett, "Dual and joint EKF for simultaneous SOC and OH estimation ", Proc. 21st Electric Vehicle Symposium (EVS21), 2005, pages 1-12.

[7] YingzhiCui, PengiianZuo, ChunyuDu, YunzhiGao, JieYang, XinqunCheng, YulinMa, GepingYin, "State of health diagnosis model for lithium ion batteries based on real-time impedance and open circuit voltage parameters identification method", Energy, Vol. 144, 1 February 2018, Pages 647-656

[8] Wenzl H, Baring-Gould I, Kaiser R, Liaw BY, Lundsager P, et al. "Life prediction of batteries for selecting the technically most suitable and cost effective battery", Journal of Power Sources, Vol. 144, Issue 2, 15 June 2005, Pages 373-384

[9] Adnan Nuhic, Jonas Bergdolt, Bernd Spier, Michael Buchholz and Klaus Dietmayer, "Battery Health Monitoring and Degradation Prognosis in Fleet Management Systems" World Electric Vehicle Journal 2018, 9, 39

[10] Ngoc-Tham Tran, Abdul Basit Khan and Woojin Choi, "State of Charge and State of Health Estimation of AGM VRLA Batteries by Employing a Dual Extended Kalman Filter and an ARX Model for Online Parameter Estimation" Energies 2017, Vol. 10, 137

[11] R. Xiong, L. Li, Z. Li, Q. Yu, H. Mu, "An electrochemical model based degradation state identification method of Lithium-ion battery for all-climate electric vehicles application”, Appl. Energy 219 (2018) 264-275.

[12] L. Zhang, L. Wang, C. Lyu, J. Li, J. Zheng, "Non-destructive analysis of degradation mechanisms in cycle-aged graphite/LiCoO2 batteries", Energies 7 (2014) 6282-6305.

[13] G.J. May, N. Maleschitz, H. Diermaier, T. Haeupl, "The optimisation of grid designs for valve-regulated lead/acid batteries for hybrid electric vehicle applications", Journal of Power Sources, Vol. 195, Issue 14, 15 July 2010, Pages 4520-4524.

[14] Z. Chen, C.C. Mi, Y. Fu, J. Xu, X. Gong, "Online battery state of health estimation based on Genetic Algorithm for electric and hybrid vehicle applications”, Journal of Power Sources, Vol. 240 (2013) Pages 184-192.

[15] J.C. Forman, S.J. Moura, J.L. Stein, H.K. Fathy, "Genetic identification and Fisher identifiability analysis of the Doyle-Fuller-Newman model from experimental cycling of a LiFePO4 cell", Journal of Power Sources 210 (2012) Pages 263-275.

[16] K. Yamada, K. Maeda, K. Sasaki, T. Hirasawa, "Computer-aided optimization of grid design for high-power leadacid batteries", Journal of Power Sources, Vol. 144, Issue 2, 15 June 2005, Pages 352-357

[17] D. Pavlov, Lead-acid Batteries Science and Technology, Elsevier, Ox-ford, 2017, ISBN- 9780444595607.

[18] H.-T. Lin, T.-J. Liang, and S.-M. Chen, "Estimation of Battery State of Health Using Probabilistic Neural Network," IEEE Trans. Ind. Informatics, vol. 9, no. 2, 2013, pp. 679-685.

[19] G.K. Prasad, C.D. Rahn, "Model based identification of aging parameters in lithium ion batteries", J. Power Sources 232 (2013) Pages 79-85.

[20] J. Li, K. Adewuyi, N. Lotfi, R.G. Landers, J. Park, “A single particle model with chemical/mechanical degradation physics for lithium ion battery State of Health (SOH) estimation”, Appl. Energy 212 (2018) 1178-1190.

[21] R. Deshpande, M. Verbrugge, Y.-T. Cheng, J. Wang, P. Liu, "Battery cycle life prediction with coupled chemical degradation and fatigue mechanics", J. Electrochem. Soc. 159 (2012) A1730-A1738.

[22] J. Wang, P. Liu, J. Hicks-Garner, E. Sherman, S. Soukiazian, M. Verbrugge, et al., "Cycle-life model for graphiteLiFePO4 cells", J. Power Sources 196 (2011) 3942-3948.

[23] M. Petit, E. Prada, V. Sauvant-Moynot, "Development of an empirical aging model for Li-ion batteries and application to assess the impact of Vehicle-to-Grid strategies on battery lifetime", Appl. Energy 172 (2016) 398407.

[24] N. Omar, M.A. Monem, Y. Firouz, J. Salminen, J. Smekens, O. Hegazy, et al., "Lithium iron phosphate based battery - assessment of the aging parameters and development of cycle life model ", Appl. Energy 113 (2014) 15751585 .

[25] M. Ecker, J.B. Gerschler, J. Vogel, S. Käbitz, F. Hust, P. Dechent, et al., "Development of a lifetime prediction model for lithium-ion batteries based on extended accelerated aging test data ", J. Power Sources 215 (2012) 248 257.

[26] Z. Li, L. Lu, M. Ouyang, Y. Xiao, "Modeling the capacity degradation of LiFePO4/ graphite batteries based on stress coupling analysis", J. Power Sources 196 (2011) 9757-9766.

[27] R.B. Wright, C.G. Motloch, J.R. Belt, J.P. Christophersen, C.D. Ho, R.A. Richardson, et al., "Calendar- and cyclelife studies of advanced technology development program generation 1 lithium-ion batteries", J. Power Sources 110 (2002) 445-470.

[28] J. Schmalstieg, S. Käbitz, M. Ecker, D.U. Sauer, "A holistic aging model for Li (NiMnCo)O2 based 18650 lithiumion batteries", J. Power Sources 257 (2014) 325-334.

[29] Baghdadi, O. Briat, J.Y. Delétage, P. Gyan, J.M. Vinassa, "Lithium battery aging model based on Dakin's degradation approach", J. Power Sources 325 (2016) 273-285.

[30] R. Xiong, J. Tian, H. Mu, C. Wang, "A systematic model-based degradation behavior recognition and health monitoring method for lithium-ion batteries", Appl. Energy 207 (2017) 372-383.

[31] R. Yang, R. Xiong, H. He, H. Mu, C. Wang, "A novel method on estimating the degradation and state of charge of lithium-ion batteries used for electrical vehicles”, Appl. Energy 207 (2017) 336-345. 


\section{BIOGRAPHIES OF AUTHORS}
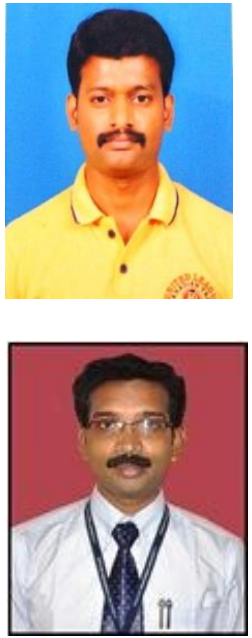

D. Selvabharathi received B.E degree in ECE from Valliammai Engg college, Affiliated to Anna University, Tamil Nadu, India in 2007, and received the M.Tech degree in Power Electronics and Drives from SRM University, Kattankulathur, Tamilnadu, India in 2012. He is currently a Research Scholar and Pursuing his research in the area of Battery Health Monitoring Systems from Periyar Maniammai Institute of Science \& Technology, Thanjavur, India. His research interest includes advancing the battery health management techniques for real time monitoring and diagnostics.

N. Muruganantham received B.E. degree in Electrical and Electronics Engineering from K.S.R College of Technology, Affiliated to Periyar University, Salem and M.E. degree in Power Electronics and Drives from Anna University, Chennai, Tamil Nadu, India. He did his research (Ph.D.) from Anna University, Chennai. He is working as Associate Professor \& Head in the Department of Electrical and Electronics Engineering, Periyar Maniammai Institute of Science \& Technology (Deemed to be University), Thanjavur, Tamil Nadu, India. He is a professional member in IEEE (Power Electronics Society), IE (Institute of Engineers) and ISTE. He has published more than 25 research papers in reputed International journals. His research interests are soft-switching converters, special electrical machines and its control, artificial intelligence, e-vehicle, transformer \& battery health monitoring systems and smart meters. 